\title{
[FAB]ricando la vivienda
}

\section{Vanessa Zadel Velásquez}

Universidad de Lima, Perú

Recibido: 22 de octubre del 2016 / Aprobado: 11 de enero del 2017

\begin{abstract}
Este artículo forma parte de mi investigación de tesis de maestría titulada Hacia una nueva pedagogía arquitectónica. Incorporación y aplicación de nuevas tecnologías en las facultades de Arquitectura. Situación actual en Lima y perspectivas generales. Busca que se reflexione sobre la fabricación digital relacionada con la vivienda y las posibilidades que de ella puedan surgir. Se presentan ejemplos de construcciones en distintos países, pues, de pronto, todo puede sentirse inmediato, cercano, instantáneo y, a la vez, efimero, casi imperceptible. La arquitectura, hoy en día, no permanece ajena a lo digital, tampoco en Lima; si bien formalmente lo digital no se muestra aún en nuestro medio construido (me refiero a la generación de formas complejas), sí lo hace como parte del proceso proyectual. ¿Qué tan lejos nos encontramos del siguiente paso?
\end{abstract}

nuevas tecnologías, fabricación digital, diseño modular, Fab Labs

\section{[FAB]ricating Housing}

This article is part of my master's thesis research entitled "Towards a new architectural pedagogy. Incorporation and application of new technologies in the Schools of Architecture. Current situation in Lima and general perspectives." The purpose is to make people reflect on the digital fabrication of housings and the possibilities that may arise therefrom, with examples from different countries because, all of a sudden, everything can be perceived immediate, close, momentary, ephemeral, and almost imperceptible. At present, architecture does not remain unconnected to digital design, neither in Lima. Even when the digital aspect is not formally shown in our built means (I refer to the generation of complex forms), it is so as a part of the project process. How far are we from the next step?

new technologies, digital fabrication, modular design, Fab Labs 


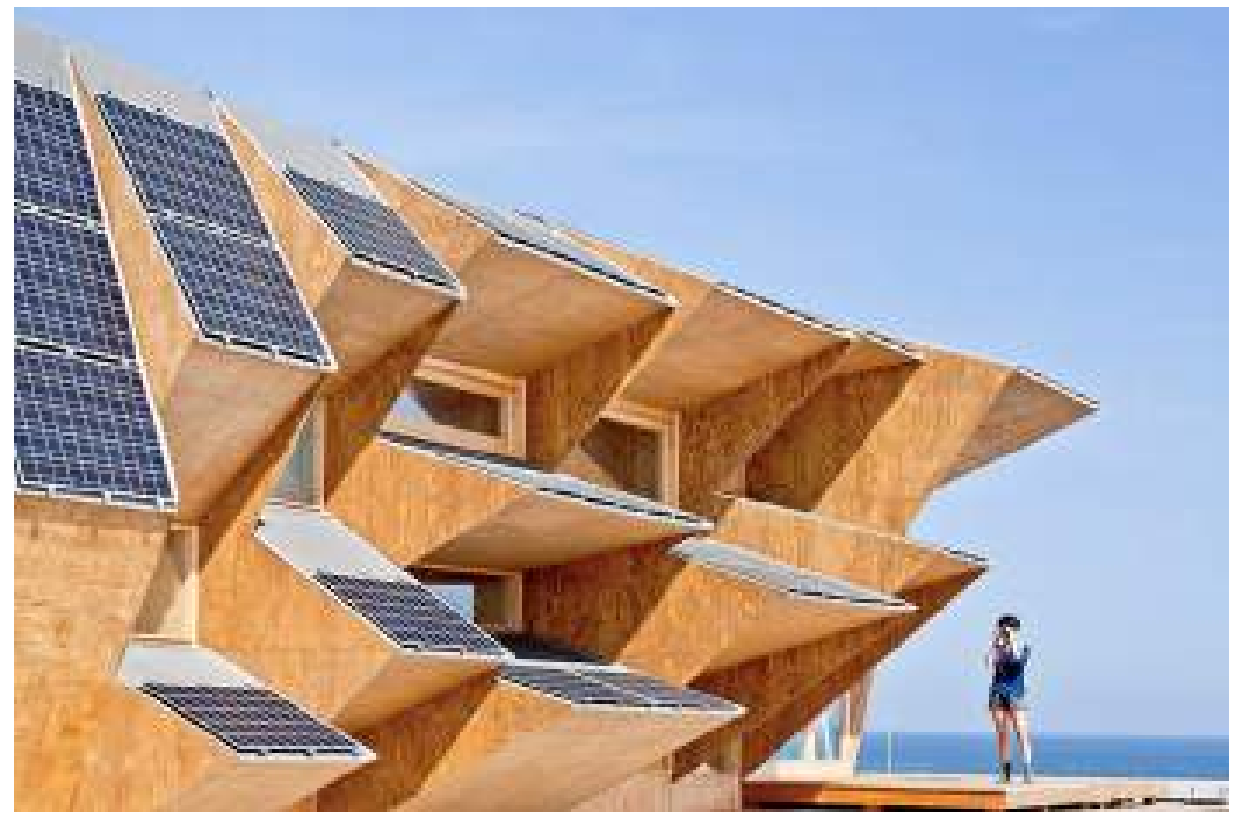

Figura 1

Endesa Pavilion Solar House 2.0

Fuente: http://inhabitat.com/barcelonas-solar-house-2-0-pavilion-built-with-modular-photovoltaic-panel-roof/

El uso de la tecnología ha cambiado los códigos de comunicación y la manera en que nos relacionamos con los demás. Nos enfrenta a cambios que se concretan cada vez más rápido, lo cual no significa que comprendamos por completo lo que está pasando. Aprendemos a vivir con dichos cambios y los asumimos como parte del contexto en el que vivimos. La arquitectura, hoy en día, no permanece ajena a la dimensión digital, no solo la utiliza como parte del proceso de diseño o como herramienta de expresión grafica, sino también como medio de fabricación a escala real.

Los procesos de fabricación digital se dan a inicio del siglo XXI con la aparición de los laboratorios de fabricación digital, conocidos también como Fab Labs. "El concepto de Fab Lab fue impulsado desde 2001 por el Center of Bits and Atoms del Massachusetts Institute of Technology (CBA-MIT) a cargo de Neil Gershenfeld, como un programa para democratizar el acceso al conocimiento y herramientas de fabricación digital” (Herrera y Juárez, 2012).

No se haría esperar la proliferación de dichos Fab Labs (Laboratorios de Fabricación Digital) principalmente en los Estados Unidos de Norte América y, posteriormente, alrededor del mundo. Pronto la red de Fab Labs tendría un crecimiento sostenible en el tiempo. Quince años después de su creación hay cerca de 1050 laboratorios de fabricación digital en todo el mundo (dato obtenido a diciembre de 2016 en la página web 
fabfoundation.org). El crecimiento de la red mundial de Fab Labs permite llegar cada vez a más lugares alejados y se vuelve accesible a una mayor cantidad de personas.

Dichos Fab Labs tienen por objetivo la exploración de soluciones mediante el diseño y la fabricación digital: desde piezas pequeñas hasta piezas modulares para la construcción prefabricada o, inclusive, se usa en medicina humana para la impresión de órganos. El alcance abarca propuestas individuales y colectivas. Es de carácter multidisciplinario, pues, según el tipo de proyecto, se reunirán distintos especialistas (arquitectos, diseñadores industriales, biólogos, etc.), a fin de participar juntos en la propuesta de diseño y posteriormente en su fabricación digital. Lo importante es que los Fab Labs no pretenden tener un acceso exclusivo al ámbito profesional, sino que buscan llegar a todo tipo de usuarios y despertar en ellos la curiosidad por crear soluciones personalizadas a determinados problemas o situaciones.

Al ser una red conectada virtualmente se puede modelar determinado objeto en Lima e imprimirlo en un Fab Lab en Japón. La distancia deja de medirse en kilómetros, lo que vuelve difusos los límites físicos. En esta red todos somos cercanos, inmediatos y atemporales. En esta red, los baby boomers, la generación X, los millennials y la generación Y coinciden, comparten, enseñan y aprenden entre ellos sin ser la edad algún tipo de factor diferenciador.

Si (casi) todo puede ser diseñado y luego fabricado utilizando nuevas tecnologías, ¿qué impide que se pueda diseñar una vivienda y pueda fabricarse digitalmente? Nada. Por más extraño que esto resulte, son situaciones que ya se vienen dando en países como China, donde la empresa Winsun imprime viviendas en concreto, o en Italia, donde la empresa Wasp ha logrado imprimir casas utilizando barro y paja. Las exploraciones inician en el ámbito académico donde se hacen pruebas escala 1:1 con materiales, módulos y tipos de ensambles que permitan tener una producción en serie y un ahorro de tiempo en la construcción y el traslado de materiales. Y el diseño desempeña un papel protagónico para lograrlo.

En nuestro país, también se vienen dando estas exploraciones, aunque, por el momento, solo quedan en la fabricación manual. Los proyectos que se desarrollan en diferentes universidades como exploraciones aún quedan en escala de instalaciones temporales o como exploraciones de modelos dentro de los talleres de diseño de algunas facultades de Arquitectura de Lima.

Estas exploraciones son igual de importantes, pues despiertan la curiosidad de futuros arquitectos por encontrar distintas maneras de solucionar un problema, incorporando la fabricación como parte del proceso y no como un factor limitante.

Por supuesto que todo cambio genera controversia y discusión, sobre todo si estos cambios se relacionan con la tecnología y el diseño:"¿Cuánto tiempo toma encontrar el producto que nos gusta? En términos de consumo, hace dos décadas encontrábamos una o dos opciones por producto. Hoy tenemos cientos de posibilidades a nuestro alcance" (Herrera y Juárez, 2012). 


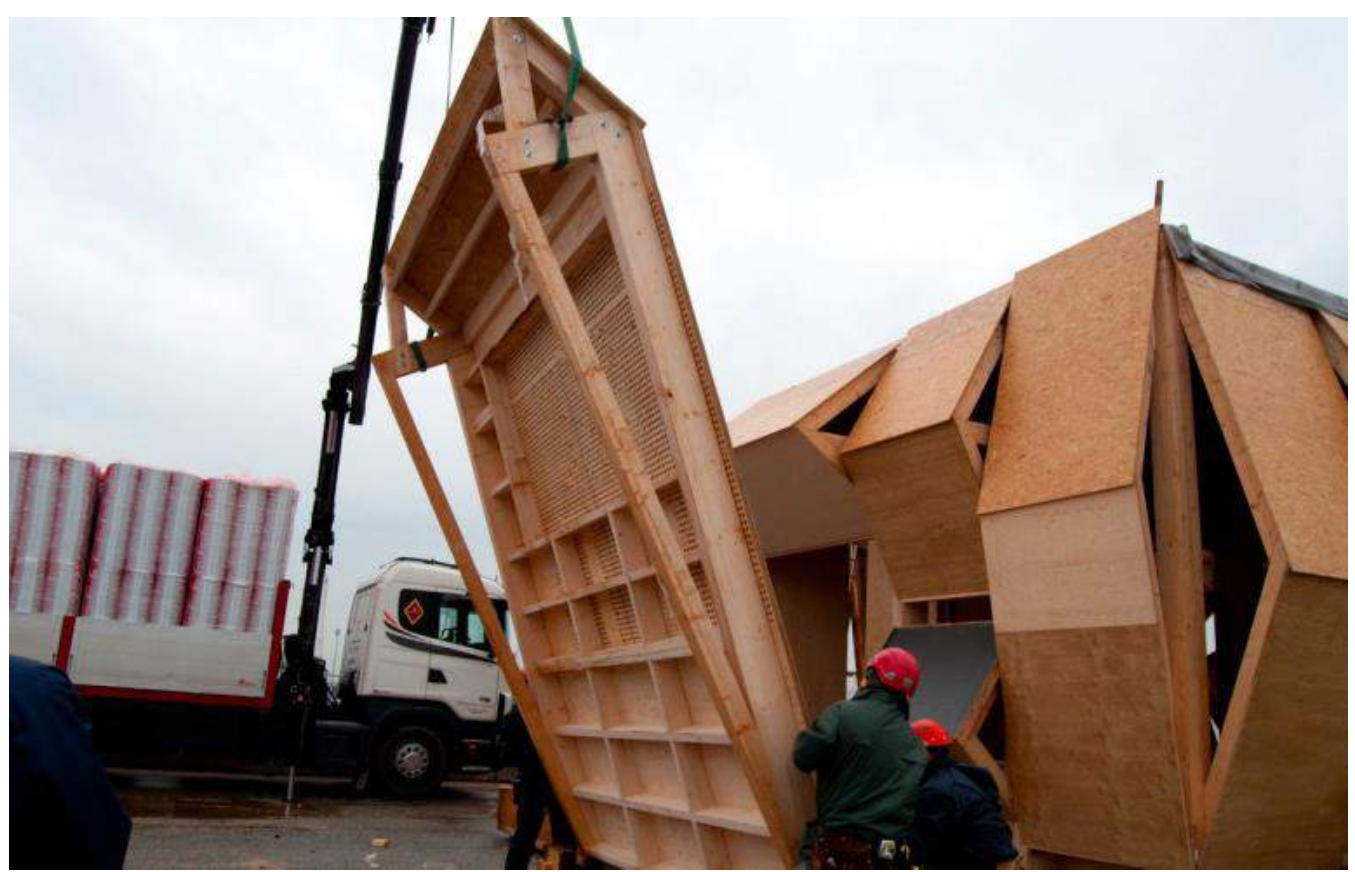

Figura 2

Construcción Endesa Pavilion Solar House 2.0

Fuente: http://inhabitat.com/barcelonas-solar-house-2-0-pavilion-built-with-modular-photovoltaic-panel-roof/

No hay nada estandarizado y si lo hay, está sujeto, por completo, a cualquier tipo de modificación o personalización, pero este es otro tipo de discusión. Deseo centrarme en la inmediatez, en la distancia del clic. Imaginemos que tenemos un banco de datos, llamémoslo "banco de soluciones de diseño". Ante cualquier eventualidad, yo podría acceder a los modelos de vivienda que este banco contiene e "imprimirlos". No solo obtendría la solución edificada en un tiempo mucho menor, pues podría ahorrar bastante en las coordinaciones con todos los intermediarios que surgen entre mi necesidad y la vivienda construida, sino que los costos de fabricación podrían reducirse considerablemente, ya que se puede considerar un tipo de ensamblaje "participativo" y utilizar materiales de la zona. Esto, por supuesto, teniendo un laboratorio de fabricación digital cerca.

"Con el desarrollo de diferentes herramientas de diseño asistido por ordenador (CAD) y otros paquetes de software, la variedad de procesos de diseño que están a disposición de los arquitectos y que pueden influir en el proyecto de la arquitectura y de sus componentes es más grande que nunca. Uno de los elementos que suscita un especial interés en este campo es la reciente capacidad de combinar técnicas y procesos analógicos y digitales para producir objetos fisicos, ya sean diagramas tridimensionales, maquetas a escala o prototipos a tamaño real” (Dunn, 2012). 


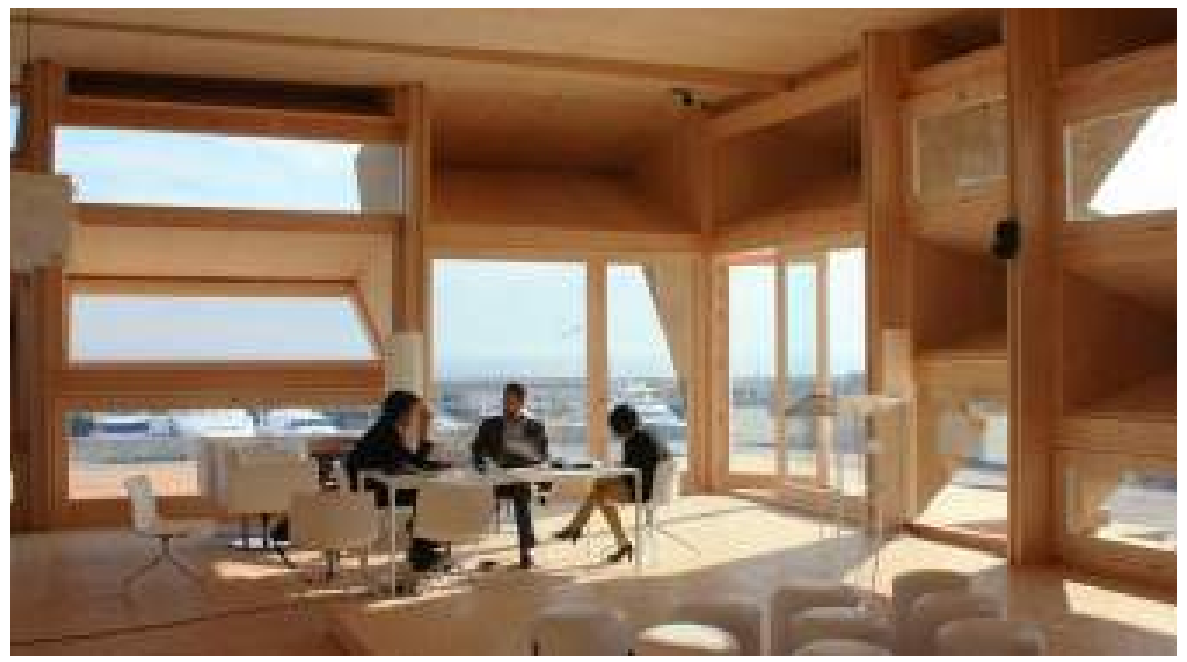

Figura 3

Interior Endesa Pavilion Solar House 2.0

Fuente: http://inhabitat.com/barcelonas-solar-house-2-0-pavilion-built-with-modular-photovoltaic-panel-roof/

El Instituto de Arquitectura Avanzada de Catalonia (IAAC) realizó la Fab House $\left(73,5 \mathrm{~m}^{2}\right)$ como propuesta en la competencia europea Decatlon Solar 2010. El proyecto ganó el premio People Choise Award, lo que respondió no solo a las exigencias de diseño establecidas en el concurso, sino que también propuso como innovación la fabricación digital como medio para optimizar recursos (materiales, económicos y temporales) e incorporar tecnologías que permitan a la vivienda producir su propia energía. "Hemos querido superar la idea de que una casa solar es una casa tradicional con paneles solares en la cubierta y mucha tecnología en su interior. Nuestro proyecto presenta una casa solar íntegra, de nueva generación que los visitantes han sabido apreciar" (FabLab House).

El proyecto siguió los procesos regulares de diseño, como la identificación del problema, la propuesta de solución, la elección de materiales, el diseño de detalles, el dibujo de planos, e incorporó el uso de la tecnología para la simulación de resultados, midiendo la eficiencia tecnológica de la construcción.

El proyecto buscaba no solo cumplir con los requerimientos de diseño, sino también aprovechar la oportunidad para dar un paso más y, mediante el uso de tecnología, lograr mejores resultados. "Hemos querido superar la idea de que una casa solar, es una casa tradicional con paneles solares en la cubierta y mucha tecnología en su interior. Nuestro proyecto presenta una casa solar íntegra, de nueva generación que los visitantes han sabido apreciar" (FabLab House).

La Fab House fue visitada por más de 20000 personas durante los diez días del concurso. 


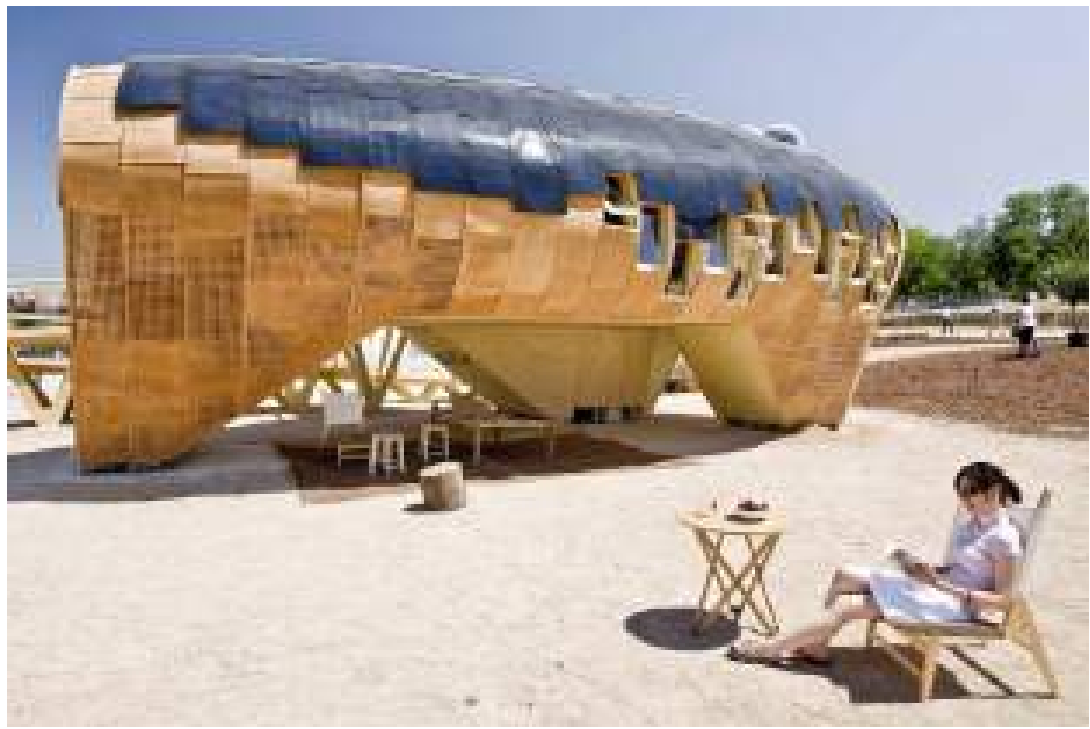

Figura 4

IAAC Fab House

Fuente: http://openbuildings.com/buildings/fab-lab-house-profile-40821

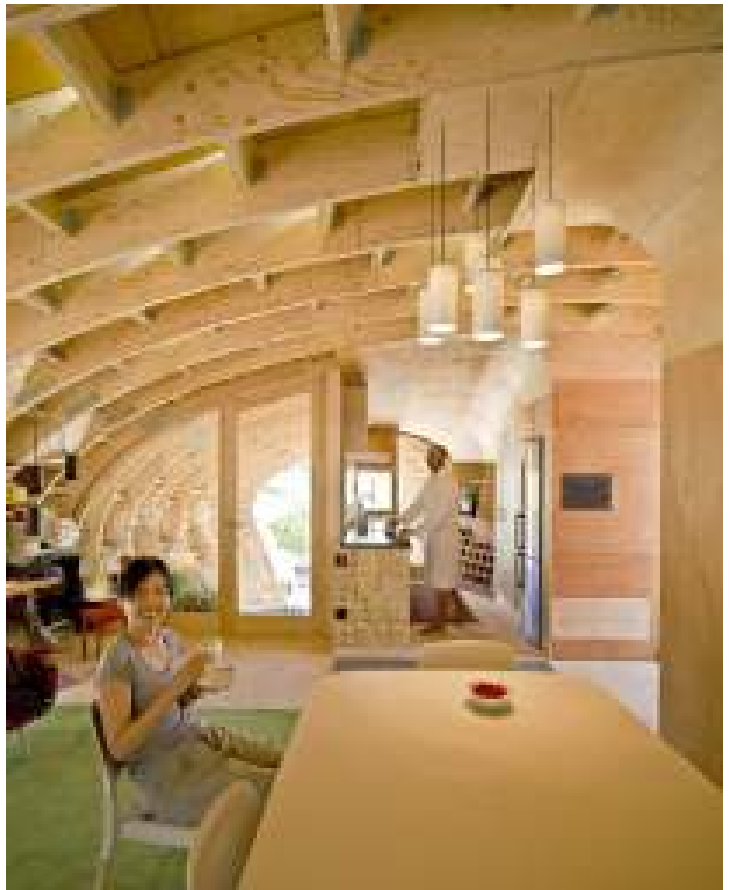

Figura 5

Fab House del IAAC

Fuente: http://openbuildings.com/buildings/

fab-lab-house-profile-40821 
La tecnología en el siglo XXI aparece vinculada a una nueva manera de crear. Actualmente, el popular DIY (do it yourself) nos pone a prueba en la producción de objetos y soluciones construibles. El proyecto Pajanachel, en Guatemala, se produce dentro de este marco de exploración y responde a un déficit de viviendas y analiza las formas de cohabitar y coproducir que se dan en cada grupo de personas, a fin de poder idear soluciones de vivienda adaptables a las necesidades de cada tipo de usuario. "Los procesos actuales y los modelos que se realizan en serie, sin un modelo adaptado a las necesidades, éstas que solo cumplen con una demanda en cuestión a compra y venta de vivienda, y permiten la concentración de vivienda en serie, como condominios y barrios cerrados, sin pensar en la habitabilidad, creando segregación social, y entonces surge de éste análisis la respuesta a re-emplear en nuestro modelo el Co-Habitar y Co-Producir" (Tiu, 2016).

El proyecto congregó a 150 alumnos de Arquitectura de Guatemala, quienes realizaron estudios de usuarios para determinar los diferentes tipos de necesidades. Diseñaron un módulo que respondió a dichas necesidades y la vivienda fue construida por sus habitantes, pues si bien las piezas fueron cortadas con láser, fueron ensambladas manualmente. "El modelo es entendible para todo mundo, sin necesidad de herramientas ni procedimientos dificiles, de una manera innovadora donde el 'hágalo usted mismo» abarca desde como emplear nuevas tecnologías de fabricación y construcción” (Tiu, 2016).

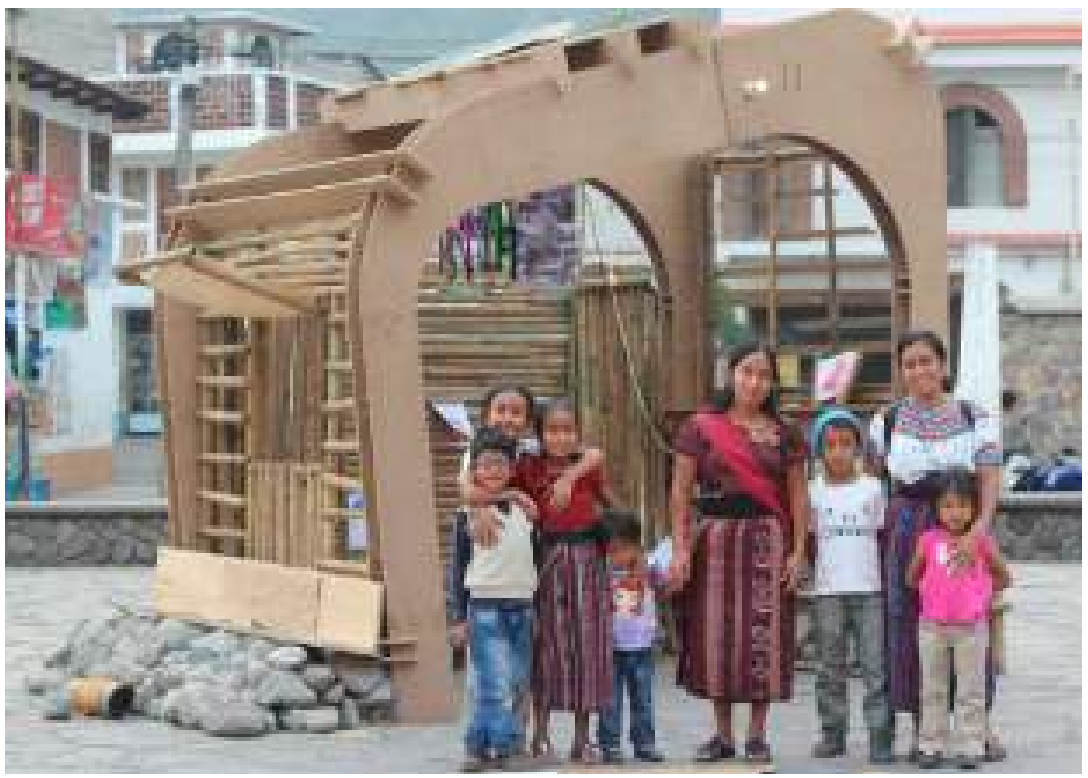

Figura 6

Proyecto Aplicado Panajachel, Guatemala

Fuente: http://criticarq.gt/articulos/academia/proyecto-fabricacion-digital-y-vivienda-productiva Fotografía: María Fernanda Palacios 


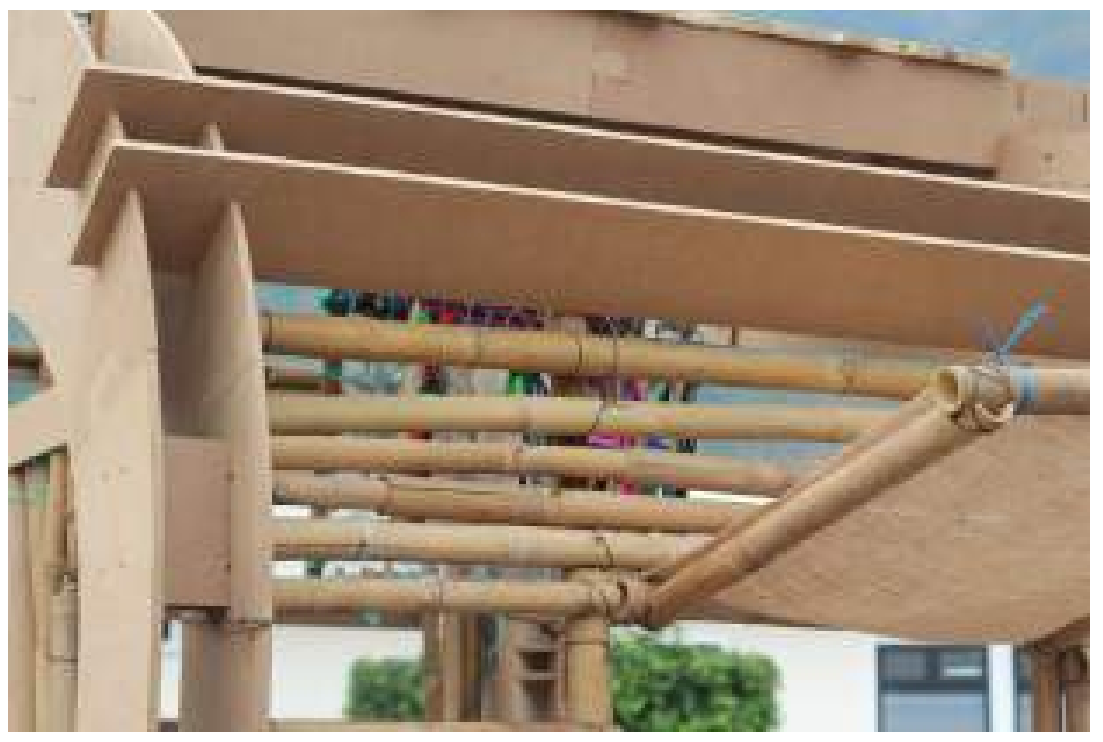

Figura 7

Armado de parte del Proyecto Aplicado Panajachel, Guatemala

Fuente: http://criticarq.gt/articulos/academia/proyecto-fabricacion-digital-y-vivienda-productiva Fotografía: María Fernanda Palacios

El proyecto Edesa World Fab Condenser, ubicado en Barcelona y construido en el 2014 es un prototipo que se hizo con motivo del BCN FAB10, Congreso Internacional en Fabricación Digital. "El prototipo explora conexiones entre diseño paramétrico, estrategias climáticas pasivas y fabricación local por control numérico" (Lab, 2014).

En dos meses se tuvo listo el scripting (programación mediante lógicas de diseño paramétrico) del prototipo, la fabricación digital en cinco días y el ensamblado en cuatro. Esto fue posible con la ayuda de voluntarios de Fab Labs de todo el mundo; razón por la cual, al hablar de este proyecto, se menciona que fue diseñado con participación internacional.
Este proyecto es un ejemplo de que el diseño paramétrico (proceso de diseño basado en algoritmos que expresan parámetros) más la fabricación digital pueden combinar esfuerzos, a fin de lograr un diseño que utilice eficientemente la tecnología y llegar a soluciones bioclimáticas que logren un máximo confort interno con poco gasto energético. Se aprovecharían, además, las condiciones climáticas del lugar y se instalarían, si fuera necesario, fuentes de energía renovable, como los paneles solares. "El Edesa World Fab Condenser es un prototipo termodinámico, una cúpula bioclimática [...]. El icosaedro original se deforma para minimizar la radiación solar incidente [...]. La piel se adapta para maximizar la ventilación natural-artificial, los vientos norte y sur (montaña y mar) inciden en momentos alternos del día" (Lab, 2014). 


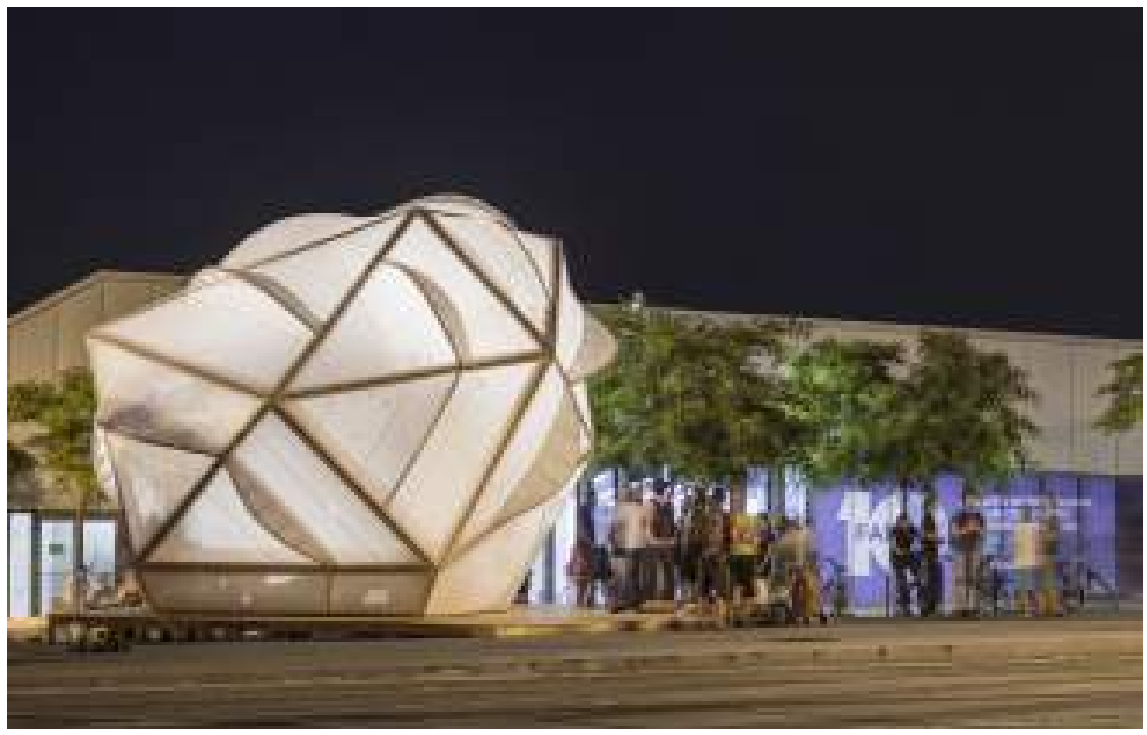

Figura 8

Endesa World Fab Condenser / MARGEN-LAB

Fuente http://www.archdaily.mx/mx/627163/endesa-world-fab-condenser-margen-lab

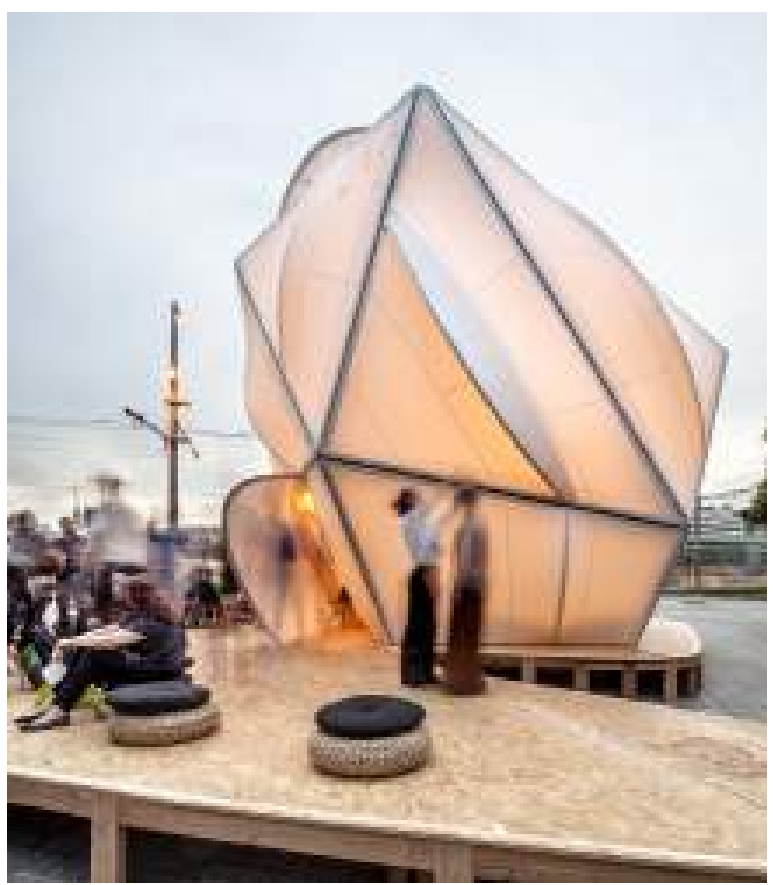

Figura 9

Endesa World Fab Condenser / MARGEN-LAB Fuente: http://www.archdaily.mx/mx/627163/ endesa-world-fab-condenser-margen-lab 
Es interesante ver las exploraciones que surgen del lado académico, como los resultados de los ejercicios colectivos realizados por alumnos en las facultades de Arquitectura en distintas partes del mundo, tanto de pregrado como de posgrado.

El pabellón Caterpillar fue construido en el 2014 por alumnos de Arquitectura e Ingeniería de la Universidad de Sevilla. "Se trata de una galería de 10 metros de largo y 3.5 de alto, construida a través de 'cáscaras' de madera 5 mm de espesor" (Franco, 2015). El proyecto exploró la madera como material y fue diseñado, modelado y fabricado digitalmente. El ensamble fue manual.

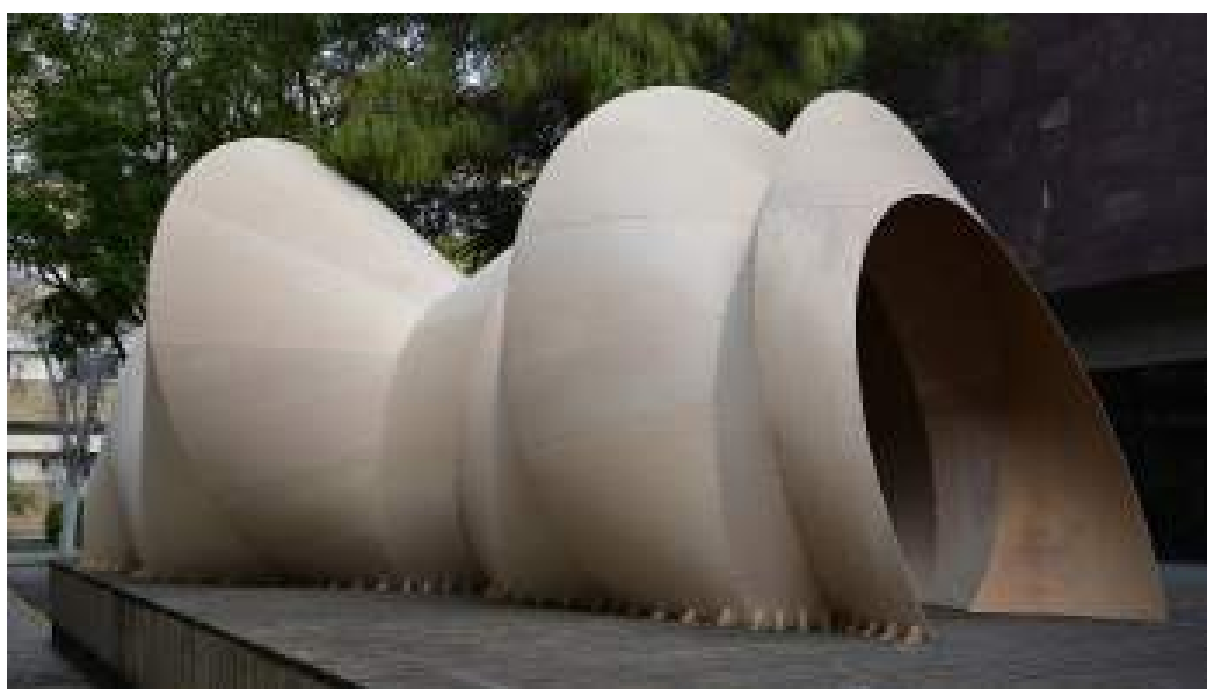

Figura 10

Pabellón Caterpillar, Universidad de Sevilla, España

Fuente: http://www.archdaily.mx/mx/760312/estudiantes-de-arquitectura-e-ingenieria-se-unen-para-construirpabellon-oruga-de-madera 


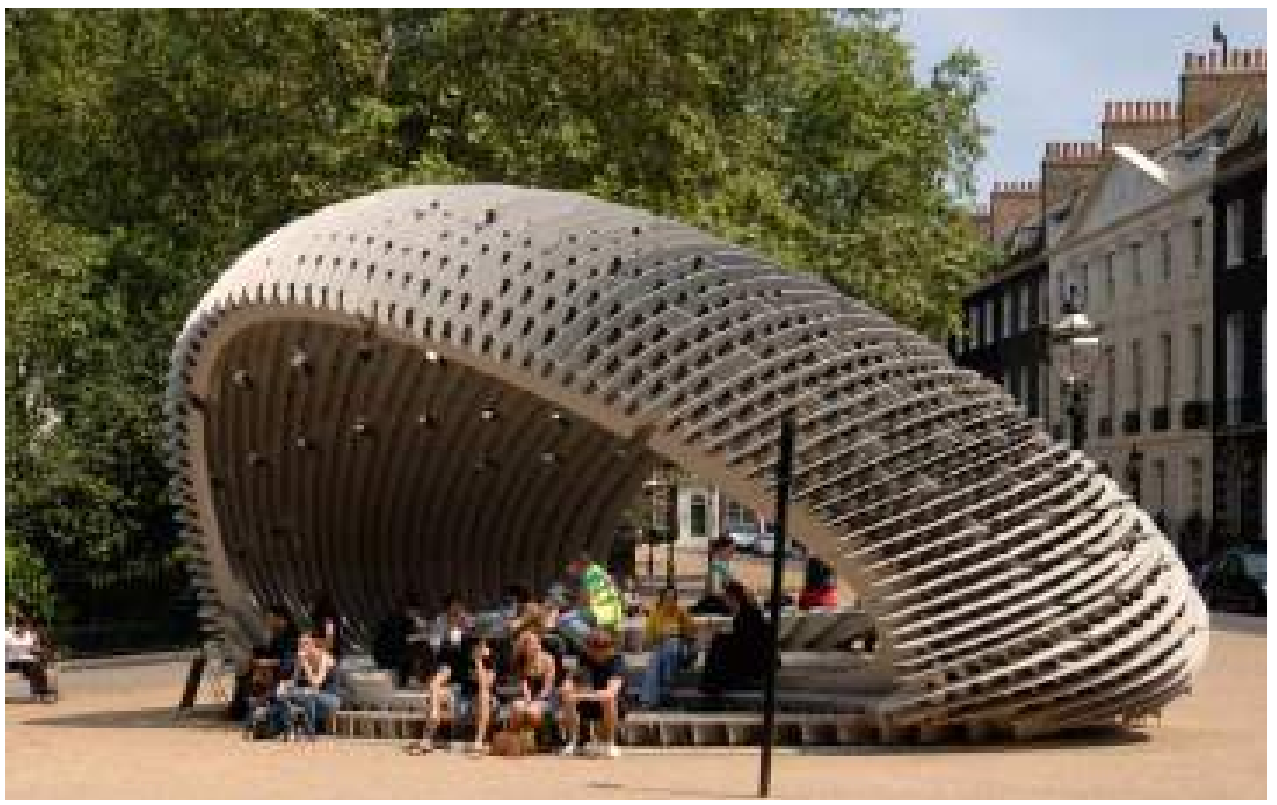

Figura 11

DRL10 Pavilion. Architectural Association

Fuente: http://www.nex-architecture.com/projects/drl10-pavilion/

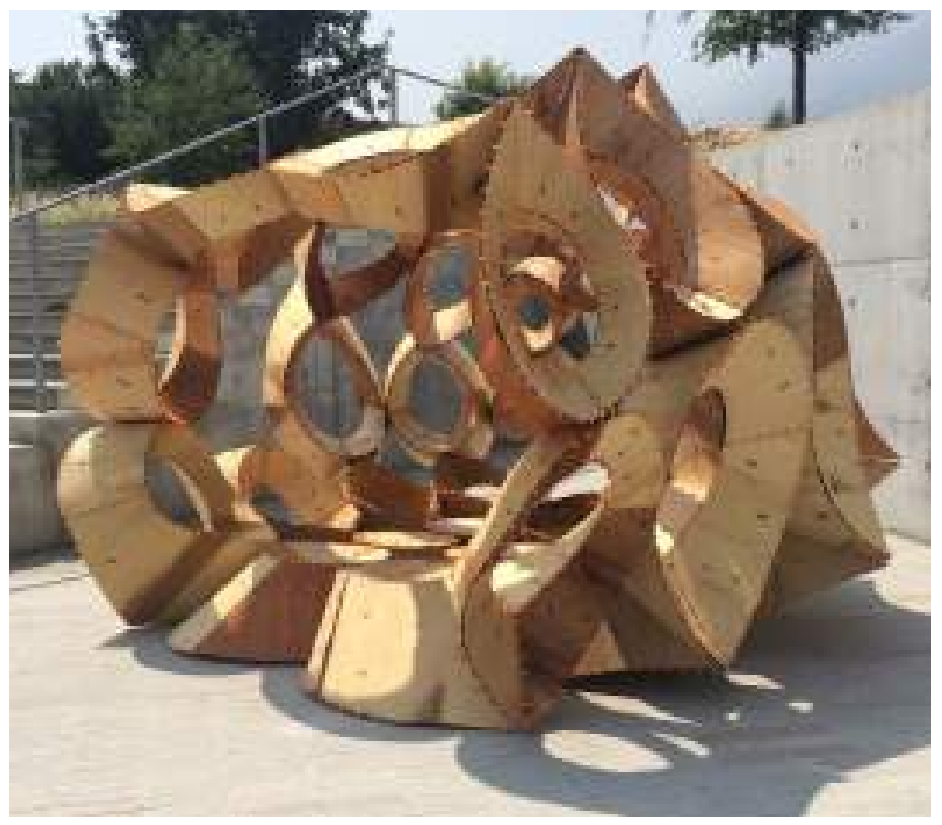

Figura 12

Bicho6. Pabellón paramétrico. Estudiantes de la UDEM

Fuente: http://www.archdaily.mx/mx/789028/ mexico-estudiantes-de-la-udem-disenan-yfabrican-bichos-una-familia-de-pabellonesparametricos 


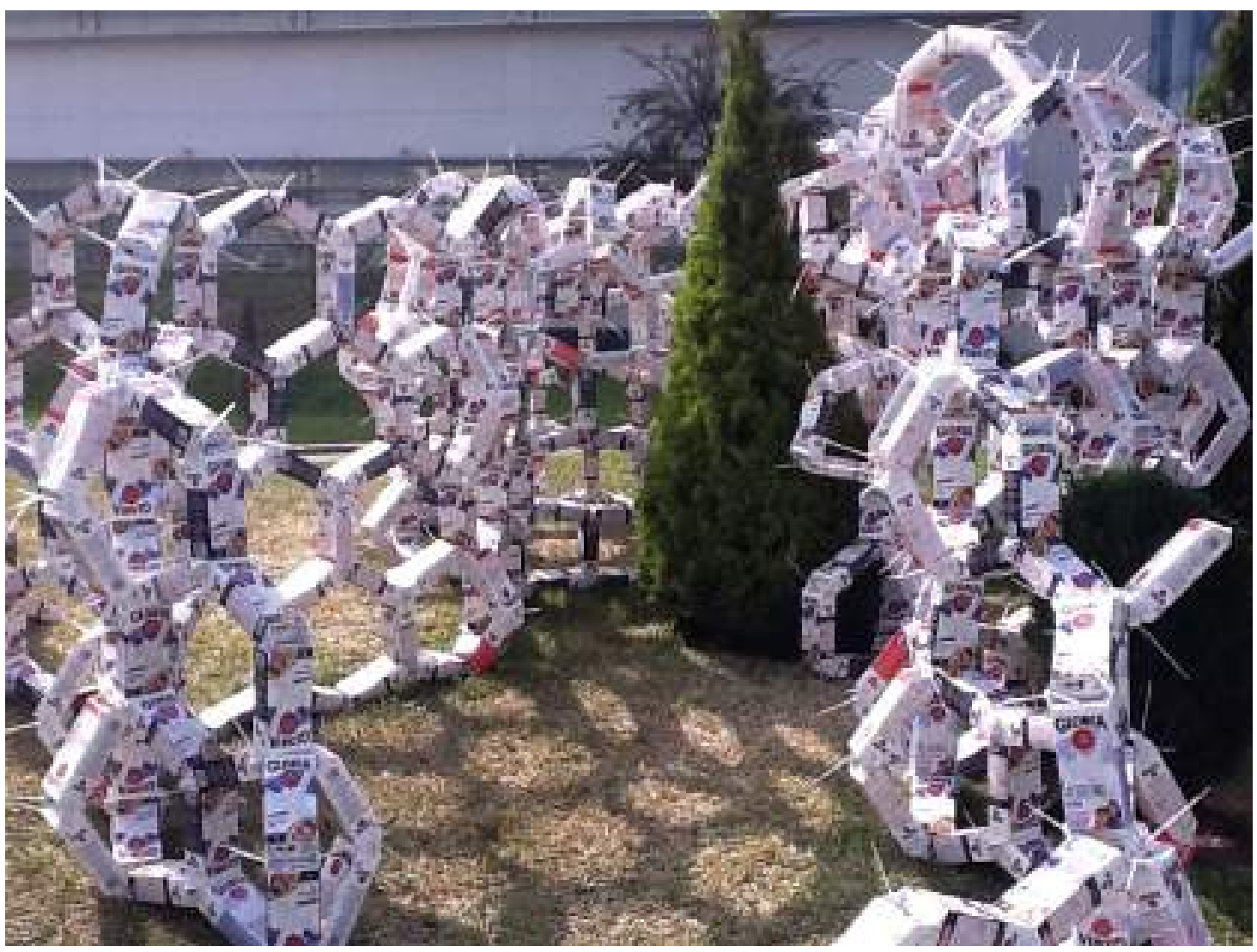

Figura 13

Proyecto Pak: Paola Aucahuasi, Jiyeon Lee, Giuliana Godoy, Alejandra Andrade Docentes del Taller Básico A: Madeleine García, Edwin Motte, Vanessa Zadel

Fotografía: Vanessa Zadel

En 2012 un grupo de alumnas del primer año de la carrera de Arquitectura de la Universidad de Lima desarrolló el proyecto Pak, como una instalación temporal, a modo de exploración formal que permitiera delimitar de una cantidad definida de espacios utilizando objetos que luego puedan ser reciclados. El resultado fue un tipo de piel adaptable y versátil: las cajas TetraPak. Estudiaron el producto con detenimiento y advirtieron cómo empalmar mejor las uniones para que sea más estable se adapte a determinada topografia, sin quebrar la continuidad de la piel, y genere patrones, aprovechando las sombras a distintas horas del día. El proyecto no involucró la fabricación digital pero permitió ver el interés de los alumnos por la exploración de nuevas soluciones y el entendimiento de determinados parámetros de diseño que pueden ser trasladados a plataformas digitales. Esta exploración sirve como estudio previo para desarrollar un proyecto complejo en un Fab Lab haciendo uso de los distintos tipos de tecnologías. 


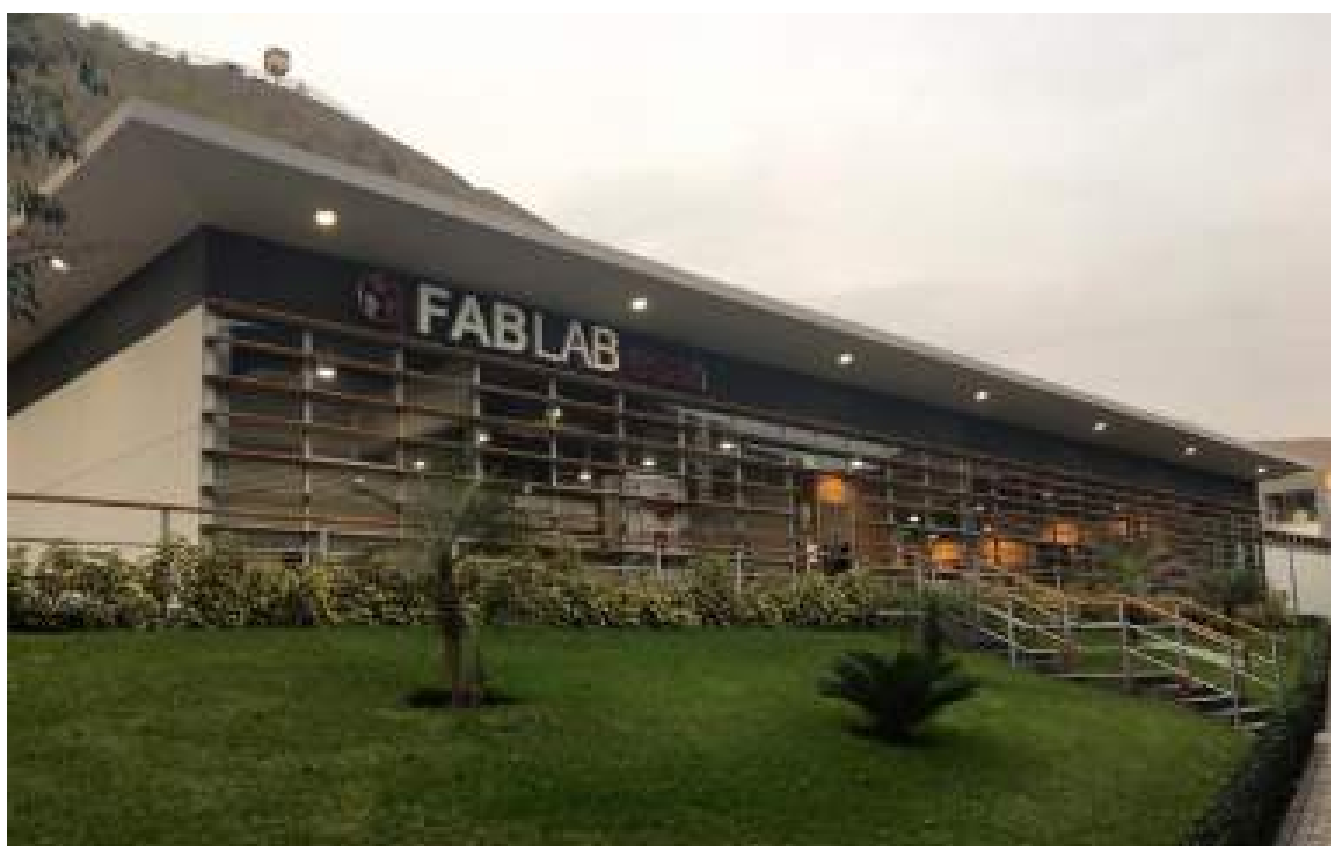

Figura 14

Fab Lab Esan. Lima, Perú

Fotografía: Arq. Vanessa Zadel

El primer Fab Lab de América del Sur fue el de la Universidad Nacional de Ingeniería (UNI) creado en el 2011 y dirigido, durante un periodo, por el arquitecto Benito Juárez y por el diseñador industrial Víctor Freundt. Este centro pertenece a la red mundial de Fab Labs liderada y guiada por la Fab Foundation. Fue implementado con la intención de ser un centro de exploración de diseño y fabricación digital y que se vuelva un detonador en el crecimiento de nuevos laboratorios de fabricación digital y que sirva, a la vez, como centro de capacitación o formador de capacitadores Fab Lab.

La aparición de Fab Labs en nuestro país se ha concentrado en la capital, Lima, donde actualmente contamos con ocho Fab Labs, un Fab café. Hay cuatro Fab Labs en proyecto, tres de los cuales se abrirán en el interior del país.

Por lo general los Fab Labs de todo el mundo están vinculados a universidades, pues es desde la academia que se da el mayor número de exploraciones. Fab Lab Buenos Aires, Fab Lab Ámsterdam, Fab Lab Lima son algunos ejemplos de los laboratorios que se crean para "democratizar el acceso a la fabricación digital y a las nuevas tecnologías de diseño, proponiendo una modalidad de trabajo colaborativa y en red, ofreciendo un ambiente favorable para la vinculación y el desarrollo integral de capacidades tecnológicas, donde la creatividad y el conocimiento dan origen a la innovación" (https://www.fablabs.io/ fablabbuenosaires). 
"Las tecnologías digitales están transformando los viejos modos de entender la prefabricación. Están cambiando los modos en los que la industria gestiona sus modos productivos. Y, lo más importante, están cambiando las formas de interrelación entre diseñador, industria y materia prima" (Lab, 2014). Pero ¿qué significa, en términos de identidad y de vínculo con el usuario, que podamos fabricar digitalmente viviendas que a su vez puedan reproducirse, imprimirse, en otras partes del mundo? En simultaneo, inclusive, si quisiéramos. ¿Estamos perdiendo identidad si imprimo una casa que fue pensada y diseñada para un lugar distinto? ¿Qué papel desempeña el arquitecto del siglo XXI si cualquier persona tiene acceso a modelos digitales que pueden ser descargados e impresos?

La tecnología ofrece las posibilidades de adaptar, modificar, transformar y ver el resultado antes de que el modelo sea construido, a fin de poder testearlo bajo criterios climáticos. Quizá nos encontremos ante un nuevo tipo de identidad: la global, y por más extraño que resulte, esa identidad global tiene la particularidad de poder adaptarse a cada uno de nosotros. Utilizamos la tecnología a diario para realizar diferentes tareas. La tecnología debe poder contribuir en generar propuestas de viviendas eficientes, climáticas, fáciles de reproducir y rápidas de ensamblar, que sean soluciones inmediatas frente a los desastres naturales. Si tuviéramos en nuestro "banco de soluciones de diseño" una serie de estructuras de viviendas modeladas que respondan a determinadas condiciones de lugar, clima y necesidades básicas, nada impediría su acceso a quienes lo necesiten.

No me refiero a la estructura de vivienda como los elementos estructurales de la ingeniería, sino como el módulo mínimo necesario en términos de cantidad de elementos que definen distintos espacios y condiciones espaciales, que permitan, por ejemplo, el aprovechamiento de determinados recursos de la zona para el cerramiento de la vivienda. La fabricación puede ser digital pero el ensamble es manual y colectivo. "Las nuevas tecnologías, generan transformaciones en la construcción del conocimiento y crisis en los modelos tradicionales para abordarlo. La coexistencia entre dos culturas, una basada a partir del texto escrito y otra multitextual, se presenta como un interesante campo de exploración" (Bessone y Mantovani, 2001).

A propósito del concurso organizado por el Colegio de Arquitectos de Lima (Concurso de Ideas Arquitectónicas "Refugios contra el friaje en zonas altoandinas"), ¿no podría ser esta una oportunidad para explorar nuevas soluciones de diseño que puedan realizarse mediante la fabricación digital y acortar así el tiempo en que se concreten dichas soluciones? Resultados que permitan la sostenibilidad de dichas viviendas, con la incorporación de elementos de energías renovables. Lograríamos no solo dar solución a un problema específico, sino que iríamos alimentando el "banco de soluciones de diseño" con propuestas que pueden reproducirse ante futuras eventualidades. [Fab]ricar la vivienda involucra muchos más que construir.

\section{CONCLUSIONES}

Las siguientes ideas se presentan para reflexión y debate:

- La fabricación digital, como proceso de producción, podría enriquecer el proceso de diseño arquitectónico en cuanto permite una ejecución más rápida de 
determinadas soluciones (a problemas ocasionados, por ejemplo, por desastres naturales).

- Los laboratorios de fabricación digital permiten a cualquier persona la exploración, personalización y producción de objetos según sus necesidades, lo cual les permite también desarrollar su creatividad.

- Los laboratorios de fabricación digital están vinculados a la sociedad donde están insertos. Esto significa que lo que se produzca responderá a lo que esa sociedad necesita. Estos requerimientos se vuelven parámetros que, sumados a los parámetros climatológicos, geográficos, culturales, etc., se logra un diseño ad hoc.

- Montar un laboratorio de fabricación digital tiene un costo económico elevado debido a la cantidad necesaria de máquinas especializadas y de personal capacitado para enseñar a utilizar dichas máquinas. Una buena alternativa es que surjan desde las universidades, lo cual permitiría la exploración desde el ámbito académico, pero con apertura al público interesado.

- Para poder fabricar digitalmente cualquier objeto se necesita modelarlo en 3D primero. Existen programas Open Source (código abierto) que se adquieren gratuita y libremente por internet.

- Existen plataformas de distribución libre como Thingiverse, de la empresa Makerbot. Estas plataformas permiten crear y compartir modelos 3D listos para su producción digital.

- Las exploraciones deberían iniciarse en el ámbito académico, pues forman a los futuros profesionales que harán uso de estas nuevas tecnologías. Las nuevas generaciones crecen viendo y probablemente usando esta tecnología, pero no necesariamente comprenden que lo que pueden lograr va más allá de cortar cartones con láser. Pueden desarrollar propuestas complejas en un menor tiempo, lo que les permitirá ayudar a más personas.

- Al permitirse el libre acceso y la descarga de modelos 3D, deben establecerse de manera clara las distintas regulaciones para definir la autoría, el uso de los objetos (que no dañen a nadie), su distribución, etcétera.

\section{REFERENCIAS}

Bessone, M., y Mantovani, G. (2000). Procesos proyectuales alternativos: en el inicio del aprendizaje del diseño arquitectonico. CumInCad. Recuperado de http://papers. cumincad.org/data/works/att/5227. content.pdf

Dunn, N. (2012). Proyecto y construcción digital en arquitectura. Barcelona: Art Blume S. L.

FabLab House.(s.f.). FabLab House. Recuperado de http://www.fablabhouse.com/la-fab-lab -house-gana-el-premio-del-publico-desolar-decathlon-europe/

Franco, J. (2015). Estudiantes de Arquitectura e Ingeniería se unen para construir "pabellón oruga" en madera. Arch Daily. Recuperado de http://www.archdaily.mx/mx/760312/ estudiantes-de-arquitectura-e-ingenieriase-unen-para-construir-pabellon-orugade-madera 
Frazer, J. (1995). An Evolutionary Architecture. Londres: Architectural Association Publications.

Herrera, P., y Juárez, B. (2012). Perspectivas en los laboratorios de fabricación digital en Latinoamérica. CuminCAD. Recuperado de http://papers.cumincad.org/data/works/att/ sigradi2012_395.content.pdf

Inhabitat. (2012). IAAC's Stunning SolarPowered Endesa Pavilion Soaks Up the Sun in Barcelona. Recuperado de http:// inhabitat.com/barcelonas-solar-house-2-0pavilion-built-with-modular-photovoltaicpanel-roof/

Institute for Advance Architecture of Catalonia, I. (s. f.). Self-Fab House. Barcelona: Actar, IaaC.
Krauel, J. (s. f.). Arquitectura digital. Innovación y diseño. Barcelona: Links.

Lab, M. (2014). Endesa World Fab Condenser. Arch Daily. Recuperado de http://www. archdaily. $\mathrm{mx} / \mathrm{mx} / 627163 /$ endesa-worldfab-condenser-margen-lab

Ribot, A., Borrego, I., García-Germán, J., y García-Setién, D. (2014). CoLaboratorio: fabricacion digital y arquitecturas colaborativas. España: Bibliografika de Voros S. A.

Tiu, V. (2016). Proyecto: Fabricación digital y vivienda productiva. Recuperado de http://criticarq.gt/articulos/academia/ proyecto-fabricacion-digital-y-viviendaproductiva 